\title{
COMPACT PERIODS OF EISENSTEIN SERIES OF ORTHOGONAL GROUPS OF RANK ONE
}

\author{
JOÃO PEDRO BOAVIDA
}

\begin{abstract}
Fix a number field $k$ with its adele ring $\mathbb{A}$. Let $G=\mathrm{O}(n+3)$ be an orthogonal group of $k$-rank 1 and $H=\mathrm{O}(n+2)$ a $k$-anisotropic subgroup. We unwind the global period

$$
\left(E_{\varphi}, F\right)_{H}=\int_{H_{k} \backslash H_{\mathbb{A}}} E_{\varphi} \cdot \bar{F}
$$

of a spherical Eisenstein series $E_{\varphi}$ of $G$ against a cuspform $F$ of $H$ into an Euler product and evaluate the local factors at odd primes.
\end{abstract}

\section{INTRODUCTION}

Fix a number field $k$ with its adele ring $\mathbb{A}$. Equip $k^{n+3}$ with a quadratic form $\langle$,$\rangle with matrix$

$$
\left(\begin{array}{lll}
1 & & \\
& * & \\
& & -1
\end{array}\right)
$$

with respect to the orthogonal decomposition $k^{n+3}=\left(k \cdot e_{+}\right) \oplus k^{n+1} \oplus\left(k \cdot e_{-}\right)$. Let $G=\mathrm{O}(n+3)$ and its subgroups act always on the right.

In the same coordinates, write $k^{n+2}=\left(k \cdot e_{+}\right) \oplus k^{n+1}$ and let $H=\mathrm{O}(n+2)$ be the fixer of $e_{-}$. Let also $\Delta$ be the discriminant of the restriction of $\langle$,$\rangle to k^{n+2}$ and let $\chi$ be the corresponding quadratic character.

We consider only the case when $k^{n+2}$ is anisotropic; in particular, $G$ has $k$-rank 1 and $H$ is $k$-anisotropic. With $e=e_{+}+e_{-}$, let $P$ be the rational parabolic stabilizing the isotropic line $k \cdot e$. Its unipotent radical $N^{P}$ is the fixer of $e$ and $\left\{e_{+}, e_{-}\right\}^{\perp}=k^{n+1}$. We choose the Levi component $M^{P}$ to be the stabilizer of $k^{n+1}$ and $\Theta=\mathrm{O}(n+1)$ to be its subgroup fixing both $e_{+}$and $e_{-}$. Therefore, $M^{P}$ has the form $\mathrm{GL}(1) \times \Theta$.

We determine the period

$$
\left(E_{\varphi}, F\right)_{H}=\int_{H_{k} \backslash H_{\mathbb{A}}} E_{\varphi} \cdot \bar{F}
$$

along $H$ of a spherical Eisenstein series $E_{\varphi}$ on $G$ against a cuspform $F$ of $H$.

Given a Hecke character $\omega: \operatorname{GL}(1) \rightarrow \mathbb{C}^{\times}$, extend it trivially to $M^{P}$, and then, by right $N^{P}$-invariance, to $\varphi_{\omega}: P_{k} \backslash P_{\mathbb{A}} \rightarrow \mathbb{C}$.

2000 Mathematics Subject Classification. Primary 11F67; Secondary 11R42, 11S40.

Key words and phrases. Eisenstein series, period, automorphic, $L$-function, orthogonal group. 
Proposition. Let $E_{\varphi}=E_{\omega, \eta}$ be the Eisenstein series obtained from $\varphi_{\omega} \cdot \eta$, where $\varphi_{\omega}$ is $\Theta$-invariant and $\eta$ is a cuspform on $\Theta$. Then

$$
\left(E_{\varphi}, F\right)_{H}=\int_{\Theta_{\mathbb{A} \backslash H_{\AA}}} \varphi_{\omega}(h) \cdot(\eta, h \cdot F)_{\Theta} \mathrm{d} h .
$$

We shall see the inner period can be simplified further, essentially reducing the original problem to the case where $\varphi_{\omega}=\varphi_{s}$ is obtained from a Hecke character with the same local parameter $s$ everywhere:

Proposition. Let $\varphi=\varphi_{s} \cdot \eta$ be as above and $\alpha=(n+1) s$. Up to correction factors at finitely many places, the period against the constant function is

$$
\begin{array}{ll}
\left(E_{\varphi}, 1\right)_{H}=\frac{(\eta, 1)_{\Theta} \cdot \operatorname{vol}\left(\Theta \backslash \Theta K^{H}\right) \cdot \zeta_{k}(\alpha-n)}{L_{k}\left(\alpha-\left\lfloor\frac{n}{2}\right\rfloor, \chi\right)}, & \text { if } n \text { is odd; } \\
\left(E_{\varphi}, 1\right)_{H}=\frac{(\eta, 1)_{\Theta} \cdot \operatorname{vol}\left(\Theta \backslash \Theta K^{H}\right) \cdot \zeta_{k}(\alpha-n)}{\zeta_{k}(2 \alpha-n) / L_{k}\left(\alpha-\frac{n}{2}, \chi\right)}, & \text { if } n \text { is even. }
\end{array}
$$

The set of bad primes consists of all archimedean primes and all factors of $2 \Delta$.

Clearly, this period is nonzero if and only if $(\eta, 1)_{\Theta} \neq 0$. Section 6 discusses $\left(E_{\varphi}, F\right)_{H}$ in the general case.

These findings fit into the Gross-Prasad conjecture on periods of $\mathrm{SO}(n+1)$ automorphic forms over $\mathrm{SO}(n)$. In the case at hand, the conjecture [10-12] predicts that a representation of $\mathrm{O}(n+2)$ occurs in a representation of $\mathrm{O}(n+3)$ if and only if the corresponding tensor product $L$-function is nonzero on $\operatorname{Re} s=\frac{1}{2}$. Ichino and Ikeda [14] discuss further details and broader context is provided in papers by Jacquet, Lapid, Offen, and/or Rogawski $[17,25,26]$, and surveyed by Gross, Reeder [13], and Jiang [20].

These same periods (called there global Shintani functions) were used by Murase and Sugano [27] to obtain and study integral expressions for standard $L$-functions of the orthogonal group.

In section 1, we unwind the period into an Euler product, obtaining

$$
\left(E_{\varphi}, F\right)_{H}=\int_{\Theta_{k} \backslash H_{\mathbb{A}}} \varphi \cdot \bar{F}=\int_{\Theta_{\mathbb{A}} \backslash H_{\mathbb{A}}} \varphi_{\omega}(h) \int_{\Theta_{k} \backslash \Theta_{\mathbb{A}}} \eta(\theta) \cdot \bar{F}(\theta h) \mathrm{d} \theta \mathrm{d} h .
$$

For several sections, we focus on $\eta=1$ and $F=1$, in which case

$$
\left(E_{\varphi}, 1\right)_{H}=\operatorname{vol}\left(\Theta_{k} \backslash \Theta_{\mathbb{A}}\right) \cdot \prod_{v} \int_{\Theta_{v} \backslash H_{v}} \varphi_{\omega, v}
$$

Because $\varphi$ is spherical, the local integral is trivial at anisotropic places. At isotropic places, the local evaluation entails a choice of local parametrization for $\Theta_{v} \backslash H_{v}$, as well as a normalization of the measure. In section 2, we consider the specifics at odd primes. Notably, at good primes it is possible to reduce the evaluation to $n=1$ or $n=2$. We articulate the full details in the subsequent sections. In section 5 , we determine the correction factors at bad odd primes. In section 6 , we resume the general case and conclude that $\left(E_{\varphi}, F\right)_{H}$ is obtained from $(\eta, F)_{\Theta}$ as well as the local integrals (obtained in the preceding sections) of $\varphi_{\omega}$ against a degenerate principal series of $H$. We show also, following Garrett [4], that the periods of a cuspform are nonzero only if, almost everywhere, the cuspform generates locally the very same sort of degenerate principal series generated by the Eisenstein 
series we consider. Finally, in the appendix, we use the same methods to evaluate (the local factors of) the constant term of $E_{\varphi}$.

Throughout, we rely on $\mathrm{O}(n+1) \supset \mathrm{O}(n)$ being a Gelfand pair, a fact conjectured by J. Bernstein and established independently by Kato, Murase, and Sugano [23] and Aizenbud, Gourevitch, and Sayag [2].

Acknowledgements. This paper is based on part of the author's doctoral dissertation, done under the supervision of Paul Garrett. It is influenced by discussions with and talks by him. The author wishes also to acknowledge the helpful advice of a referee.

\section{Contents}

Introduction

1. Setup

2. Odd primes

3. Good odd primes $-n=2 k+1$

4. Good odd primes $-n=2 k+2$

5. Bad odd primes

6. The general case

Appendix A. The constant term

References

\section{Setup}

Recall we fixed a number field $k$ with adele ring $\mathbb{A}$, and a quadratic form $\langle$, with matrix

$$
\left(\begin{array}{lll}
1 & & \\
& * & \\
& & -1
\end{array}\right)
$$

with respect to the decomposition $k^{n+3}=\left(k \cdot e_{+}\right) \oplus k^{n+2} \oplus\left(k \cdot e_{-}\right)$. We set $e=e_{+}+e_{-}$and named the following groups of isometries:

$$
\begin{aligned}
G & =\mathrm{O}(n+3), & & \text { the isometry group of }(* * *) ; \\
H & =\mathrm{O}(n+2), & & \text { the isometry group of }(* *) ; \\
\Theta & =\mathrm{O}(n+1), & & \text { the isometry group of }(*) ; \\
P & \subset G, & & \text { the } k \text {-parabolic stabilizing } k \cdot e ; \\
N^{P} & \subset P, & & \text { its unipotent radical, fixing } e \text { and }(*) ; \\
M^{P} & \subset P, & & \text { the Levi component stabilizing }(*) .
\end{aligned}
$$

The modular function of $P$ is given by $\delta_{P}(p)=|t|^{n+1}$ when $e \cdot p=e / t$. In particular, $\delta_{P}^{s}(p)=|t|^{\alpha}$, with $\alpha=(n+1) s$.

Recall $M^{P}$ has the form GL(1) $\times \Theta$. Given a Hecke character $\omega: k^{\times} \backslash \mathbb{J} \rightarrow \mathbb{C}^{\times}$, extend it trivially to $M^{P}$, and then, by right $N^{P}$-invariance, to $\varphi_{\omega}: P_{k} \backslash P_{\mathbb{A}} \rightarrow \mathbb{C}$. Given also a cuspform $\eta: \Theta_{k} \backslash \Theta_{\mathbb{A}} \rightarrow \mathbb{C}$ normalized by $\eta(1)=1$ and spherical, define $\varphi: P_{k} \backslash P_{\mathbb{A}} \rightarrow \mathbb{C}$ by $\varphi(\theta g)=\eta(\theta) \cdot \varphi_{\omega}(g)$, where $\theta \in \Theta$ and $g \in \mathrm{GL}(1) \cdot N^{P}$. 
Let

$$
E_{\varphi}(g)=E_{\omega, \eta}(g)=\sum_{\gamma \in P_{k} \backslash G_{k}} \varphi(\gamma g) .
$$

be the spherical Eisenstein series of $G$ associated with $\varphi$, and let $F$ be a cuspform of $H$. We evaluate the period

$$
\left(E_{\varphi}, F\right)_{H}=\int_{H_{k} \backslash H_{\mathrm{A}}} E_{\varphi} \cdot \bar{F}=\int_{H_{k} \backslash H_{\AA}} \sum_{\gamma \in P_{k} \backslash G_{k}} \varphi(\gamma h) \cdot \bar{F}(h) \mathrm{d} h .
$$

By Witt's lemma, $P_{k} \backslash G_{k}$ is the space of isotropic lines in $k^{n+3}$ and $H_{k}$ acts transitively on that space (here we rely on the $k$-Witt index being 1 , so that no $k$-isotropic lines are orthogonal to $\left.e_{-}\right)$. Because $\Theta=H \cap P=\mathrm{O}(n+1)$ is the fixer of $e_{+}$and $e_{-}$,

$$
\left(E_{\varphi}, F\right)_{H}=\int_{H_{k} \backslash H_{\mathbb{A}}} \sum_{\gamma \in \Theta_{k} \backslash H_{k}} \varphi(\gamma h) \cdot \bar{F}(h) \mathrm{d} h=\int_{\Theta_{k} \backslash H_{\mathbb{A}}} \varphi \cdot \bar{F} .
$$

As $\varphi(\theta g)=\eta(\theta) \cdot \varphi_{\omega}(\theta g)$ and $\varphi_{\omega}$ is left $\Theta$-invariant,

$$
\left(E_{\varphi}, F\right)_{H}=\int_{\Theta_{\mathbb{A}} \backslash H_{\mathbb{A}}} \varphi_{\omega}(h) \int_{\Theta_{k} \backslash \Theta_{\mathbb{A}}} \eta(\theta) \cdot \bar{F}(\theta h) \mathrm{d} \theta \mathrm{d} h .
$$

Because $H \supset \Theta$ is a Gelfand pair [2] and $\eta$ is spherical, the inner integral can be expressed as

$$
\int_{\Theta_{k} \backslash \Theta_{\mathbb{A}}} \eta(\theta) \cdot \bar{F}(\theta h) \mathrm{d} \theta=(\eta, F)_{\Theta} \cdot f(h),
$$

where $f$ is a spherical vector of $\operatorname{Ind}_{\Theta}^{H} 1$ normalized by $f(1)=1$. Therefore,

$$
\left(E_{\varphi}, F\right)_{H}=(\eta, F)_{\Theta} \cdot \int_{\Theta_{\mathbb{A}} \backslash H_{\mathbb{A}}} \varphi_{\omega} \cdot f .
$$

From now on and until section 6 , we restrict our attention to $\eta=1$ and $F=1$ (thus, also $\varphi=\varphi_{\omega}$ and $f=1$ ) and work locally. Therefore, we omit mention of the place $v$ whenever possible.

Anisotropic places. At anisotropic places, the local integral is $\operatorname{vol}(\Theta \backslash H)$. (Recall we are dealing with spherical functions only.)

Isotropic places. Choose a hyperbolic pair $x, x^{\prime}$ in $k^{n+2}$ so that $e_{+} \in k \cdot\left\{x, x^{\prime}\right\}$ and change coordinates so that the restricted quadratic form has matrix

$$
\left(\begin{array}{lll} 
& & 1 \\
& B &
\end{array}\right)
$$

with respect to the orthogonal decomposition $k^{n+2}=\left(k \cdot x^{\prime}\right) \oplus k^{n} \oplus(k \cdot x)$. Recall that $H=\mathrm{O}(n+2)$ and that $\Theta=\mathrm{O}(n+1)$ is the subgroup of $H$ fixing $e_{+}$. We are to evaluate

$$
\int_{\Theta \backslash H} \varphi .
$$

Let $Q \subset H$ be the parabolic stabilizing the line $k \cdot x$; we claim that $\Theta Q$ is the open orbit of $\Theta \backslash H / Q$. By Witt's lemma, $\Theta \backslash H$ can be identified with the homogeneous space of vectors $y$ with $\langle y, y\rangle=1$ and the orbit of $e_{+}$under the action of $Q$ includes all such $y$ not orthogonal to $x$ : the only non-trivial requirement is that $\langle y q, x q\rangle=$ $\langle y, x\rangle$, which can be achieved by choosing $x q=\mu x$ and $\langle y, x\rangle=\langle y q, x q\rangle=\mu\langle y q, x\rangle$. 
Therefore,

$$
\int_{\Theta \backslash H} \text { function }(h) \mathrm{d} h=\int_{\Theta \backslash \Theta Q} \text { function }(q) \mathrm{d} q=\int_{(\Theta \cap Q) \backslash Q} \text { function }(q) \mathrm{d} q .
$$

Here, $\Theta \cap Q=\mathrm{O}(n)$ is the fixer of $x$ and $x^{\prime}$. Set

$$
m_{\lambda}=\left(\begin{array}{lll}
\lambda & & \\
& \text { id } & \\
& & 1 / \lambda
\end{array}\right) \quad \text { and } \quad n_{a}=\left(\begin{array}{ccc}
1 & a & -\frac{1}{2} B(a) \\
& \text { id } & * \\
& & 1
\end{array}\right) .
$$

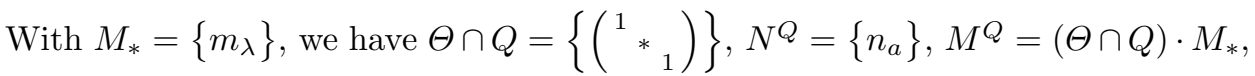
and $Q=M^{Q} \cdot N^{Q}$. The elements of $(\Theta \cap Q) \backslash Q$ can be expressed as $n_{a} \cdot m_{\lambda}$ and $\delta_{Q}\left(m_{\lambda}\right)=|\lambda|^{n}$. Moreover,

$$
\mathrm{d}\left(n_{a} \cdot m_{\lambda}\right)=\mathrm{d} a \mathrm{~d} \lambda
$$

(with $\mathrm{d} \lambda$ multiplicative and $\mathrm{d} a$ additive) is a right-invariant measure.

From this point onward, we must consider each place separately.

\section{ODD PRIMES}

Fix (and suppress) an odd place $v$. Choose coordinates so that the maximal compact open subgroup $K$ of $H$ consists of the orthogonal matrices with integral entries. With $\Phi$ being the characteristic function of $\mathfrak{o}^{n+3}$ and $\omega$ a character of $k^{\times}$, define, for this section only,

$$
\varphi(g)=\int_{k^{\times}} \omega(t) \cdot \Phi(t e \cdot g) \mathrm{d} t \quad \text { and } \quad \varphi^{\circ}=\frac{\varphi}{\varphi(1)} .
$$

Note that this is a change in notation: what was denoted $\varphi_{\omega}$ in the last section, will be denoted $\varphi^{\circ}$ in this one. We address the case $\omega=|\cdot|^{\alpha}$.

We set notation for the next several sections. Recall that $\alpha=(n+1) s$. The discriminant enters the picture as $\varepsilon=\chi(\Delta)$, where $\chi$ is the non-trivial character on $\mathfrak{o}^{\times} / \mathfrak{o}^{\times 2}$ and $\widetilde{k}=k[x] /\left\langle x^{2}-\Delta\right\rangle$ is the quadratic extension. At bad primes (those dividing the discriminant), we set $\varepsilon=0$. We write

$$
Z(\alpha)=\int_{k^{\times} \cap \mathfrak{o}}|t|^{\alpha} \mathrm{d} t=\frac{1}{1-q^{-\alpha}} \quad \text { and } \quad L(\alpha, \chi)=\frac{1}{1-\varepsilon q^{-\alpha}} .
$$

We write also

$$
Z_{\widetilde{k} / k}(\alpha)=Z(\alpha) L(\alpha, \chi) .
$$

We set $|t|=q^{-T}$ and $a=q^{-\alpha}$ throughout. We shall use

$$
\int_{k \times \cap \mathfrak{o}}|t|^{\alpha} u^{T} \mathrm{~d} t=\int_{k^{\times} \cap \mathfrak{o}}(a u)^{T} \mathrm{~d} t=\sum_{T \geq 0}(a u)^{T}=\frac{1}{1-a u}=Z\left(\alpha-\log _{q} u\right) .
$$

In this section, we express the local integral

$$
\int_{\Theta \backslash H} \varphi^{\circ}
$$

in terms of the functions $X$ and $\Pi$ that we are about to define. We prove also it is sufficient to evaluate those functions (and the corresponding local integrals) for small $n$. 
Definition. With $z=q^{-\beta}$, we define

$$
\begin{aligned}
X_{\ell}^{B}(\rho) & =\operatorname{meas}\left\{a \in \mathfrak{o}^{n}: \frac{B(a)-\rho}{2}=0 \bmod \varpi^{\ell}\right\} ; \\
X^{B}(\beta ; \rho) & =\sum_{\ell \geq 0} z^{\ell} X_{\ell}^{B}(\rho) ; \quad \text { and } \\
\Pi^{B}(\alpha, \beta) & =\int_{k^{\times} \cap \mathfrak{o}}|t|^{\alpha} X^{B}\left(\beta ; t^{2}\right) \mathrm{d} t .
\end{aligned}
$$

(When there is no risk of ambiguity, we suppress $B$ or $\rho$, or use $n$ instead of $B$.)

(3) Proposition. The local integral is obtained from

$$
\int_{\Theta \backslash H} \varphi^{\circ}=\frac{\operatorname{vol}(\Theta \backslash \Theta K) \cdot \Pi^{n}(\alpha-\beta-n, \beta)}{Z(\alpha) \cdot X^{n}(0 ; 1)}
$$

with $\beta=0$.

Proof. To account for the normalization implied in the integral (2), we consider also the integral of $\Phi(e \cdot h)$, which happens to be, at odd primes, the characteristic function of $\Theta \backslash \Theta K$. (Indeed, for $h \in H$, both or neither of $e \cdot h$ and $e^{\prime} \cdot h$ are in $\mathfrak{o}^{n+3}$. The statement follows at once from the Cartan decomposition in $G$.)

That allows us to express the local integral (still without $|\lambda|^{\beta}$ ) as

$$
\operatorname{vol}(\Theta \backslash \Theta K) \cdot \frac{\int_{\Theta \backslash H} \varphi^{\circ}}{\int_{\Theta \backslash H} \operatorname{ch}_{\Theta \backslash \Theta K}}=\frac{\operatorname{vol}(\Theta \backslash \Theta K) \cdot \int_{(\Theta \cap Q) \backslash Q} \varphi}{\varphi(1) \cdot \int_{(\Theta \cap Q) \backslash Q} \operatorname{ch}_{\Theta \backslash \Theta K}} .
$$

With a new parameter $\beta=0$, the integral in the numerator of (4) is

$$
\int_{(\Theta \cap Q) \backslash Q} \varphi \cdot|\lambda|^{\beta}=\int_{k^{\times}} \int_{k^{\times}} \int_{k^{n}}|t|^{\alpha}|\lambda|^{\beta} \Phi\left(t e \cdot n_{a} \cdot m_{\lambda}\right) \mathrm{d} a \mathrm{~d} \lambda \mathrm{d} t .
$$

Recall that we chose coordinates so that $e_{+}$be a linear combination of $x$ and $x^{\prime}$. We specify further that $e_{+}=x^{\prime}+\frac{1}{2} x$. Noting that

$$
e \cdot n_{a} \cdot m_{\lambda}=\left(e_{+}+e_{-}\right) \cdot n_{a} \cdot m_{\lambda}=e_{+} \cdot n_{a} \cdot m_{\lambda}+e_{-},
$$

we have (in $k^{n+2}$ )

$$
e_{+} \cdot n_{a} \cdot m_{\lambda}=\left(\begin{array}{lll}
1 & 0 & \frac{1}{2}
\end{array}\right) \cdot n_{a} \cdot m_{\lambda}=\left(\begin{array}{lll}
\lambda & a & \frac{1}{2 \lambda}(1-B(a)
\end{array}\right)
$$

and (in $k^{n+3}$ )

$$
t e \cdot n_{a} \cdot m_{\lambda}=\left(\lambda t, a t, \frac{1}{2 \lambda t}\left(t^{2}-B(a t)\right), t\right) .
$$

Therefore, continuing (5) and after a change of variables,

$$
\begin{aligned}
\int_{(\Theta \cap Q) \backslash Q} \varphi \cdot|\lambda|^{\beta} & =\int_{k^{\times}} \int_{k^{\times}} \int_{k^{n}}|t|^{\alpha-\beta-n}|\lambda|^{\beta} \Phi\left(\lambda, a, \frac{1}{2 \lambda}\left(t^{2}-B(a)\right), t\right) \mathrm{d} a \mathrm{~d} \lambda \mathrm{d} t \\
& =\int_{k^{\times} \cap \mathfrak{o}}|t|^{\alpha-\beta-n} \int_{k^{\times} \cap \mathfrak{o}}|\lambda|^{\beta} \int_{\mathfrak{o}^{n}} \operatorname{ch}_{\mathfrak{o}}\left(\frac{t^{2}-B(a)}{2 \lambda}\right) \mathrm{d} a \mathrm{~d} \lambda \mathrm{d} t .
\end{aligned}
$$

Consider now the integral in the denominator of (4). We have

$$
\begin{aligned}
\int_{(\Theta \cap Q) \backslash Q} \operatorname{ch}_{\Theta \backslash \Theta K} & =\int_{k^{\times}} \int_{k^{n}} \Phi\left(e \cdot n_{a} \cdot m_{\lambda}\right) \mathrm{d} a \mathrm{~d} \lambda \\
& =\int_{k^{\times}} \int_{k^{n}} \Phi\left(\lambda, a, \frac{1}{2 \lambda}(1-B(a)), 1\right) \mathrm{d} a \mathrm{~d} \lambda=X(0 ; 1) .
\end{aligned}
$$


Dimension reduction. By taking hyperbolic planes away, we can simplify the evaluation of (3) significantly. The argument is valid at even primes too.

We write vectors in $k^{n^{\prime}}=k^{n+2}$ as $a^{\prime}=(x, a, y)$ and the quadratic form as $B^{\prime}\left(a^{\prime}\right)=B(a)-2 x y$, where $a \in k^{n}$ and $B(a)$ is the restriction to $k^{n}$. Note that $B^{\prime}$ and $B$ have the same discriminant. Also, we abbreviate $X_{\ell}^{B^{\prime}}(\rho)=X_{\ell}^{\prime}(\rho)$, $X^{B^{\prime}}(\beta ; \rho)=X^{\prime}(\beta ; \rho), X_{\ell}^{B}(\rho)=X_{\ell}(\rho)$, and $X^{B}(\beta ; \rho)=X(\beta ; \rho)$. In particular,

$$
\begin{aligned}
& X_{\ell}^{\prime}=\operatorname{meas}\left\{(x, a, y) \in \mathfrak{o}^{n+2}: \frac{B(a)-\rho}{2}-x y=0 \bmod \varpi^{\ell}\right\} \\
& X_{\ell}=\operatorname{meas}\left\{a \in \mathfrak{o}^{n}: \frac{B(a)-\rho}{2}=0 \bmod \varpi^{\ell}\right\} .
\end{aligned}
$$

(6) Proposition. With $z=q^{-\beta}$, we have

$$
X^{\prime}(\beta ; \rho)=\frac{Z(\beta+1)}{Z(\beta+2)} \cdot X(\beta+1 ; \rho) .
$$

Moreover, if there is a hyperbolic subspace with dimension $2 k$ and $n=m+2 k$,

$$
\begin{aligned}
& X^{m+2 k}(\beta ; \rho)=\frac{Z(\beta+1)}{Z(\beta+k+1)} \cdot X^{m}(\beta+k ; \rho), \\
& \Pi^{m+2 k}(\alpha, \beta)=\frac{Z(\beta+1)}{Z(\beta+k+1)} \cdot \Pi^{m}(\alpha, \beta+k),
\end{aligned}
$$

and the local integral is

$$
\frac{\operatorname{vol}(\Theta \backslash \Theta K) \cdot \Pi^{m}(\alpha-\beta-n, \beta+k)}{Z(\alpha) \cdot X^{m}(k ; 1)} .
$$

Proof. If $\rho^{(2)}=(B(a)-\rho) / 2 \bmod \varpi^{\ell}$, then the set

$$
\left\{a \in \mathfrak{o}^{n}: \frac{B(a)-\rho}{2}-x y=0 \bmod \varpi^{\ell}\right\}
$$

breaks into

$$
\bigcup_{N \geq 0}\left\{a \in \mathfrak{o}^{n}: \operatorname{ord} \rho^{(2)}=N ; \rho^{(2)}-x y=0 \bmod \varpi^{\ell}\right\} .
$$

We shall prove in proposition (14) that, for an isotropic plane, $X_{\ell}^{2}\left(2 \rho^{(2)}\right)$ depends only on ord $\rho^{(2)}$ (we express it thus so that the argument be valid at even primes too). That allows us to write

$$
X_{\ell}^{\prime}(\rho)=\sum_{N \geq 0}\left(X_{N}(\rho)-X_{N+1}(\rho)\right) \cdot X_{\ell}^{2}\left(2 \varpi^{N}\right)
$$

and

$$
X^{\prime}(\beta ; \rho)=\sum_{\ell \geq 0} z^{\ell} X_{\ell}^{\prime}(\rho)=\sum_{N \geq 0}\left(X_{N}-X_{N+1}\right) \cdot X^{2}\left(2 \varpi^{N}\right) .
$$

Relying on proposition (14) (which, for the isotropic case and even primes is about $\left.X^{2}\left(2 \varpi^{N}\right)\right)$ and setting $w=z q^{-1}$, we obtain

$$
X^{\prime}(\beta ; \rho)=\frac{Z(\beta+1)}{Z(\beta+2)} \cdot X(\beta+1 ; \rho) .
$$


Strategy overview. The main task left is to determine $X\left(\beta ; t^{2}\right)$ and $\Pi(\alpha, \beta)$ for anisotropic forms. It will prove convenient to use $z=q^{-\beta}$ and $a=q^{-\alpha}$ consistently. We will also use $w=z q^{-1}$, as well as $u=q^{-2 \beta-m}$ (with $m$ being the dimension of the anisotropic space under consideration), so that when $\beta$ is set to the Witt index (of $k^{n}$ ) we obtain $u=q^{-n}$.

According to propositions (3) and (6), if $n=m+2 k$ (where $k$ denotes now the Witt index) the local integral is

$$
\begin{aligned}
& \frac{\operatorname{vol}(\Theta \backslash \Theta K) \cdot \Pi^{n}(\alpha-\beta-n, \beta)}{Z(\alpha) \cdot X^{n}(0 ; 1)} \\
& \quad=\frac{\operatorname{vol}(\Theta \backslash \Theta K) Z(\beta+1) Z(k+1) \Pi^{m}(\alpha-\beta-n, \beta+k)}{Z(\alpha) Z(\beta+k+1) Z(1) X^{m}(k ; 1)} .
\end{aligned}
$$

In particular, for $\beta=0$ (which is what we need for the period), we obtain

$$
\frac{\operatorname{vol}(\Theta \backslash \Theta K) \cdot \Pi^{m}(\alpha-n, k)}{Z(\alpha) \cdot X^{m}(k ; 1)} .
$$

We shall find that $X^{m}\left(\beta ; t^{2}\right)$ is a rational function of $z$ (which we will often write also with $w$ or $u$ ), whose dependence on $t^{2}$ is in fact a dependence on $T=\operatorname{ord} t$ only. Moreover,

$$
\Pi^{m}(\alpha, \beta)=\int_{k^{\times} \cap \mathfrak{o}}|t|^{\alpha} X^{m}\left(\beta ; t^{2}\right) \mathrm{d} t=\sum_{T \geq 0} a^{T} X^{m}\left(\beta ; \varpi^{2 T}\right) .
$$

This will be a rational function $R(z, w, u, a$ ) of $z$ (or $w$ or $u$ ) and $a$, whose factors all have the form $1 \pm q^{*}$ after $z, w, u$ and $a$ are substituted (and $a$ occurs only with non-negative exponents). At $a=0$, we obtain the coefficient of $T=0$. That is, $X^{m}(\beta ; 1)=R(z, w, u, 0)$ consists of the factors of $R$ that do not involve $a$.

In other words, if $R^{*}(z, w, u, a)$ consists of the factors of $R(z, w, u, a)$ that do involve $a$, then

$$
\frac{\Pi^{m}(\alpha, \beta)}{X^{m}(\beta ; 1)}=R^{*}(z, w, u, a) .
$$

That is, the local period is

$$
\operatorname{vol}(\Theta \backslash \Theta K) \cdot \frac{R^{*}\left(q^{-k}, q^{-k-1}, q^{-n}, q^{-(\alpha-n)}\right)}{Z(\alpha)} .
$$

(We will often suppress $\operatorname{vol}(\Theta \backslash \Theta K)$ from this point onward.)

In the prototypical scenario, we might obtain an expression like

$$
X\left(\beta ; t^{2}\right)=\frac{\mathrm{fn}(z, w, u)}{1-u}\left[1-\frac{u-v}{1-v} u^{T}\right]
$$

(where $v$ might be $w, \varepsilon w q^{-1},-z$, or a similar term). Then

$$
\Pi(\alpha, \beta)=\frac{\mathrm{fn}(z, w, u)}{1-u}\left[\frac{1}{1-a}-\frac{u-v}{(1-v)(1-a u)}\right]=\frac{\mathrm{fn}(z, w, u)(1-a v)}{(1-v)(1-a)(1-a u)} .
$$

In light of what we said above, the local period (7) would then be

$$
\frac{1}{Z(\alpha)} \cdot \frac{1-a v}{(1-a)(1-a u)}=\frac{Z(\alpha-n) Z(\alpha)}{Z(\alpha)} \cdot(1-a v)=\frac{Z(\alpha-n)}{1 /(1-a v)} .
$$




\section{GOOD ODD PRIMES $-n=2 k+1$}

We consider first $n=1$. The quadratic form on $k^{1}$ can be expressed as $\Delta x^{2}$.

(10) Proposition. With $z=q^{-\beta}, w=z q^{-1}, u=z^{2} q^{-1}$, and $\Delta$ a unit, we have

$$
X^{1}\left(\beta ; t^{2}\right)=\frac{1+w}{1-u}\left[1-\frac{u-\varepsilon w}{1-\varepsilon w} u^{T}\right] .
$$

Proof. We first evaluate

$$
X_{\ell}\left(t^{2}\right)=\operatorname{meas}\left\{x \in \mathfrak{o}^{1}: \Delta x^{2}=t^{2} \bmod \varpi^{\ell}\right\} .
$$

If $t^{2}=0 \bmod \varpi^{\ell}$ (i.e., if $\ell \leq 2 T$ ), we have

$$
X_{\ell}=X_{\ell}(0)=\operatorname{meas}\left\{x \in \mathfrak{o}: x^{2}=0 \bmod \varpi^{\ell}\right\}=q^{-\lceil\ell / 2\rceil}
$$

and

$$
\sum_{0 \leq \ell \leq 2 T} z^{\ell} q^{-\lceil\ell / 2\rceil}=1+\sum_{0<k \leq T}\left(z^{-1}+1\right) z^{2 k} q^{-k}=\frac{1+w}{1-u}-\frac{w+u}{1-u} u^{T} .
$$

On the other hand, if $t^{2} \neq 0 \bmod \varpi^{\ell}$ (i.e., if $\ell>2 T$ ), we have two distinct situations: either $\Delta x^{2}-t^{2}$ is isotropic $(\varepsilon=1)$ or it isn't $(\varepsilon=-1)$.

When $\varepsilon=-1$, the form $\Delta x^{2}-t^{2}$ is anisotropic. In that case, we show in lemma (12) below that $\Delta x^{2}-t^{2}=0 \bmod \varpi^{\ell}$ requires $t^{2}=0 \bmod \varpi^{\ell}$. Therefore, $X_{\ell}=0$ for $t^{2} \neq 0 \bmod \varpi^{\ell}$.

When $\varepsilon=1$, we have

$$
\Delta x^{2}-t^{2}=0 \bmod \varpi^{\ell} \Longrightarrow x^{2}=t^{2} \bmod \varpi^{\ell} \Longrightarrow|x|=|t|=q^{-T},
$$

leading to $(x-t)(x+t)=0 \bmod \varpi^{\ell}$ and $x= \pm t \bmod \varpi^{\ell-T}$. That is,

$$
X_{\ell}=\operatorname{meas}\left\{x \in \mathfrak{o}: x= \pm t \bmod \varpi^{\ell-T}\right\}=2 q^{-\ell+T}
$$

and

$$
\sum_{\ell>2 T} z^{\ell} X_{\ell}=\sum_{\ell>2 T} z^{\ell} 2 q^{-\ell+T}=\frac{2 z^{2 T+1} q^{-T-1}}{1-z q^{-1}}=\frac{2 z q^{-1}\left(z^{2} q^{-1}\right)^{T}}{1-z q^{-1}}=\frac{(1+\varepsilon) w u^{T}}{1-w} .
$$

Taking this last expression as well as (11) into account, and combining the cases $\varepsilon= \pm 1$, we obtain the result stated.

(12) Lemma. If $k$ is the local field at an odd prime and the form $B(x)=\sum a_{i} x_{i}^{2}$ is anisotropic, then

$$
|B(x)|=\max \left\{\left|a_{i} x_{i}^{2}\right|\right\} .
$$

Proof. If some coordinate had $\left|a_{i} x_{i}^{2}\right|>|B(x)|$, then Hensel's lemma would yield a new vector $x^{\prime}$ with ord $x_{i}=\operatorname{ord} x_{i}^{\prime}$ and $B\left(x^{\prime}\right)=0$.

(13) Proposition. If $\Delta$ is a unit and $n=2 k+1$, the local period is

$$
\operatorname{vol}(\Theta \backslash \Theta K) \cdot \frac{Z(\alpha-n)}{L(\alpha-k, \chi)}=\operatorname{vol}(\Theta \backslash \Theta K) \cdot \frac{Z((n+1)(s-1)+1)}{L\left((n+1)\left(s-\frac{1}{2}\right)+1, \chi\right)} .
$$


Proof. Let $z=q^{-\beta}, w=z q^{-1}, u=z^{2} q^{-1}$ and $a=q^{-\alpha}$. In proposition (10), we saw $X^{1}$ fits the prototype (8) with $v=\varepsilon w$. According to (9), the local period is

$$
\frac{Z(\alpha-n)}{1 /(1-\varepsilon a w)}=\frac{Z(\alpha-n)}{L(\alpha-n+k+1, \chi)} .
$$

The result follows from $\alpha=(n+1) s$.

\section{GOOD ODD PRIMES- $n=2 k+2$}

We consider $n=2$ first.

Expressing the form in terms of $x$ and $y$, we may assume $B=x^{2}-\Delta y^{2}$ and note that $X_{\ell}^{B}(\rho)$ depends only on ord $\rho$. Indeed, any unit can be expressed in the form $\eta=a^{2}-\Delta b^{2}$ (this is a consequence of Hensel's lemma). But

$$
(a x+\Delta b y)^{2}-\Delta(b x+a y)^{2}=\left(a^{2}-\Delta b^{2}\right)\left(x^{2}-\Delta y^{2}\right)=\eta\left(x^{2}-\Delta y^{2}\right)
$$

and the matrix $\left(\begin{array}{cc}a & \Delta b \\ b & a\end{array}\right)$ is invertible in $\mathfrak{o}^{2}$ if its determinant $a^{2}-\Delta b^{2}$ is a unit.

(14) Proposition. With $z=q^{-\beta}, w=z q^{-1}, u=z^{2} q^{-2}$, and $\Delta$ a unit, we have

$$
X^{2}\left(\beta ; t^{2}\right)=\frac{\left(1-\varepsilon w q^{-1}\right)\left(1-\varepsilon w u^{T}\right)}{(1-w)(1-\varepsilon w)} .
$$

Proof of proposition (14) in anisotropic case. This is the case $\varepsilon=-1$. We assumed the form is $x^{2}-\Delta y^{2}$. We evaluate

$$
X_{\ell}\left(t^{2}\right)=\operatorname{meas}\left\{(x, y) \in \mathfrak{o}^{2}: x^{2}-\Delta y^{2}=t^{2} \bmod \varpi^{\ell}\right\},
$$

for $|t|=q^{-T}$.

When $t^{2}=0 \bmod \varpi^{\ell}$ (i.e., $\ell \leq 2 T$ ), the anisotropy lemma (12) leads to

$$
X_{\ell}\left(t^{2}\right)=\operatorname{meas}\left\{(x, y) \in \mathfrak{o}^{2}: x^{2}=y^{2}=0 \bmod \varpi^{\ell}\right\}=\left(q^{-\lceil\ell / 2\rceil}\right)^{2}=q^{-2\lceil\ell / 2\rceil} .
$$

We saw in (11) (using $q^{-2}, w q^{-1}=z q^{-2}$, and $u=z^{2} q^{-2}$ here in place of $q^{-1}$, $w=z q^{-1}$, and $u=z^{2} q^{-1}$ there) that

$$
\sum_{0 \leq \ell \leq 2 T} z^{\ell} X_{\ell}=\sum_{0 \leq \ell \leq 2 T} z^{\ell} q^{-2\lceil\ell / 2\rceil}=\frac{1+w q^{-1}}{1-u}-\frac{w q^{-1}+u}{1-u} u^{T} .
$$

When $t^{2} \neq 0 \bmod \varpi^{\ell}$ (i.e., $\ell>2 T$ ), again by anisotropy,

$$
X_{\ell}\left(t^{2}\right)=\operatorname{meas}\left\{(x, y) \in \mathfrak{o}^{2}: x^{2}-\Delta y^{2}=t^{2} \bmod \varpi^{\ell}\right\}=|t|^{2} \cdot X_{\ell-2 T}(1) .
$$

For each fixed $\ell$,

$$
\begin{aligned}
\sum_{\text {unit } \eta \bmod \varpi^{\ell}} X_{\ell}(\eta) & =\sum_{\text {unit } \eta \bmod \varpi^{\ell}} \operatorname{meas}\left\{(x, y) \in \mathfrak{o}^{2}: x^{2}-\Delta y^{2}=\eta \bmod \varpi^{\ell}\right\} \\
& =\operatorname{meas}\left\{(x, y) \in \mathfrak{o}^{2}: x^{2}-\Delta y^{2}=\text { unit }\right\}=1-q^{-2} .
\end{aligned}
$$

(We relied on anisotropy for the last step.) Because $X_{\ell}(\eta)=X_{\ell}(1)$, we see that

$$
X_{\ell}(1)=\frac{1-q^{-2}}{\#\left\{\operatorname{units} \eta \bmod \varpi^{\ell}\right\}}=\left(1+q^{-1}\right) q^{-\ell}
$$

and also

$$
X_{\ell}\left(t^{2}\right)=q^{-2 T} \cdot X_{\ell-2 T}(1)=\left(1+q^{-1}\right) q^{-\ell}
$$


This leads to

$$
\sum_{\ell>2 T} z^{\ell} X_{\ell}=\sum_{\ell>2 T} w^{\ell}\left(1+q^{-1}\right)=\frac{w+w q^{-1}}{1-w} u^{T}
$$

Combining the two subsums (over $\ell \leq 2 T$ and over $\ell>2 T$ ) we obtain the stated conclusion for the anisotropic case.

Proof of proposition (14) in isotropic case. This is the case $\varepsilon=1$. We can assume the form is $x y$. We will evaluate

$$
X_{\ell}(\rho)=\operatorname{meas}\left\{(x, y) \in \mathfrak{o}^{2}: x y=\rho \bmod \varpi^{\ell}\right\} .
$$

We set $N=\operatorname{ord} \rho$.

If $\rho=0 \bmod \varpi^{\ell}$ (i.e., $\ell \leq N$ ), we have

$$
\begin{aligned}
X_{\ell} & =\operatorname{meas}\left\{x=0 \bmod \varpi^{\ell}\right\}+\sum_{0 \leq k<\ell} \operatorname{meas}\left\{\operatorname{ord} x=k ; y=0 \bmod \varpi^{\ell-k}\right\} \\
& =q^{-\ell}+\sum_{0 \leq k<\ell} q^{-k}\left(1-q^{-1}\right) q^{-\ell+k}=q^{-\ell}+\ell q^{-\ell}\left(1-q^{-1}\right)
\end{aligned}
$$

and

$$
\sum_{0 \leq \ell \leq N} z^{\ell} X_{\ell}=\sum_{0 \leq \ell \leq N}\left(w^{\ell}+\ell w^{\ell}\left(1-q^{-1}\right)\right)=\frac{1-w^{N+1}}{1-w}+\left(1-q^{-1}\right) \sum_{0 \leq \ell \leq N} \ell w^{\ell} .
$$

We observe in passing that

$$
\sum_{0 \leq \ell \leq N} \ell w^{\ell}=w\left(\sum_{0 \leq \ell \leq N} w^{\ell}\right)^{\prime}=\frac{w+(N w-N-1) w^{N+1}}{(1-w)^{2}} .
$$

Therefore,

$$
\sum_{0 \leq \ell \leq N} z^{\ell} X_{\ell}=\frac{1-w q^{-1}}{(1-w)^{2}}-\left[\frac{1}{1-w}+\frac{N\left(1-q^{-1}\right)}{1-w}+\frac{1-q^{-1}}{(1-w)^{2}}\right] w^{N+1} .
$$

If $\rho \neq 0 \bmod \varpi^{\ell}$ (i.e., $\ell>N$ ), we have

$$
\begin{aligned}
X_{\ell} & =\operatorname{meas}\left\{(x, y) \in \mathfrak{o}^{2}: x y=\rho \bmod \varpi^{\ell}\right\} \\
& =\sum_{0 \leq k \leq N} \operatorname{meas}\left\{\operatorname{ord} x=k ; y=\rho / x \bmod \varpi^{\ell-k}\right\} \\
& =\sum_{0 \leq k \leq N} q^{-k}\left(1-q^{-1}\right) q^{-\ell+k}=(N+1)\left(1-q^{-1}\right) q^{-\ell}
\end{aligned}
$$

and

$$
\sum_{\ell>N} z^{\ell} X_{\ell}=(N+1)\left(1-q^{-1}\right) \sum_{\ell>N} w^{\ell}=\frac{(N+1)\left(1-q^{-1}\right) w^{N+1}}{1-w} .
$$

After combining the two subsums (over $\ell \leq N$ and over $\ell>N$ ), the choice $\rho=t^{2}$ and $N=2 T$ completes the proof of the isotropic case. 
(15) Proposition. If $\Delta$ is a unit and $n=2 k+2$, the local period is

$$
\begin{aligned}
\operatorname{vol}(\Theta \backslash \Theta K) \cdot & \frac{Z(\alpha-n) L(\alpha-k-1, \chi)}{Z(2 \alpha-n)} \\
& =\operatorname{vol}(\Theta \backslash \Theta K) \cdot \frac{Z((n+1)(s-1)+1) L\left((n+1)\left(s-\frac{1}{2}\right)+\frac{1}{2}, \chi\right)}{Z((n+1)(2 s-1)+1)} .
\end{aligned}
$$

Proof. Let $z=q^{-\beta}, w=z q^{-1}, u=z^{2} q^{-2}$, and $a=q^{-\alpha}$. Using $X^{2}$ from proposition (14), we obtain

$$
\Pi^{2}(\alpha, \beta)=\frac{1-\varepsilon w q^{-1}}{(1-w)(1-\varepsilon w)}\left[\frac{1}{1-a}-\frac{\varepsilon w}{1-a u}\right]=\frac{\left(1-\varepsilon w q^{-1}\right)(1+\varepsilon a w)}{(1-w)(1-a)(1-a u)} .
$$

Therefore, the local period (7) is

$$
\frac{1+\varepsilon a w}{Z(\alpha) \cdot(1-a)(1-a u)}=\frac{Z(\alpha-n) L(\alpha-n+k+1, \chi)}{Z(2 \alpha-2 n+2 k+2)}
$$

and the result follows from $\alpha=(n+1) s$.

\section{BAD ODD PRIMES}

We now consider the odd primes dividing the discriminant. We describe their periods in terms of correction factors to those obtained in propositions (13) and (15). In light of the discussion leading up to (7), we need only consider anisotropic forms.

We assume the form $B$ is $B_{0}(x)+\varpi B_{1}(y)$ for $(x, y) \in \mathfrak{o}^{n_{0}} \times \mathfrak{o}^{n_{1}}$, with each $B_{*}$ integral, regular, diagonal, and anisotropic. We want to evaluate

$$
X_{\ell}^{B}\left(t^{2}\right)=\operatorname{meas}\left\{x \in \mathfrak{o}^{n_{0}}, y \in \mathfrak{o}^{n_{1}}: B_{0}(x)+\varpi B_{1}(y)=t^{2} \bmod \varpi^{\ell}\right\} .
$$

If $t^{2}=0 \bmod \varpi^{\ell}$, the anisotropy lemma (12) tells us that

$$
\begin{aligned}
X_{\ell}^{B}(0) & =\operatorname{meas}\left\{x \in \mathfrak{o}^{n_{0}}, y \in \mathfrak{o}^{n_{1}}: B_{0}(x)=\varpi B_{1}(y)=0 \bmod \varpi^{\ell}\right\} \\
& =\operatorname{meas}\left\{x \in \mathfrak{o}^{n_{0}}: B_{0}(x) \in \varpi^{\ell} \mathfrak{o}\right\} \cdot \operatorname{meas}\left\{y \in \mathfrak{o}^{n_{1}}: B_{1}(y) \in \varpi^{\ell-1} \mathfrak{o}\right\} \\
& =q^{-n_{0}\lceil\ell / 2\rceil} \cdot q^{-n_{1}\lceil(\ell-1) / 2\rceil} .
\end{aligned}
$$

If $t^{2} \neq 0 \bmod \varpi^{\ell}$, the anisotropy lemma, Hensel's lemma, and the fact that $X_{\ell}^{B_{0}}\left(t^{2}\right)$ depends only on $T=$ ord $t$, tell us that

$$
\begin{aligned}
X_{\ell}^{B}\left(t^{2}\right) & =\operatorname{meas}\left\{x \in \mathfrak{o}^{n_{0}}, y \in \mathfrak{o}^{n_{1}}: B_{0}(x)+\varpi B_{1}(y)=t^{2} \bmod \varpi^{\ell}\right\} \\
& =\operatorname{meas}\left\{x \in \mathfrak{o}^{n_{0}}, y \in \mathfrak{o}^{n_{1}}: B_{0}(x)=t^{2} \bmod \varpi^{\ell} ;\left|B_{1}(y)\right| \leq\left|B_{0}(x)\right|\right\} \\
& =\operatorname{meas}\left\{x \in \mathfrak{o}^{n_{0}}: B_{0}(x)=t^{2} \bmod \varpi^{\ell}\right\} \cdot q^{-n_{1} T}=X_{\ell}^{B_{0}}\left(t^{2}\right) \cdot q^{-n_{1} T}
\end{aligned}
$$

(If $n_{0}=0$, then the equation is $\varpi B_{1}(y)=t^{2} \bmod \varpi^{\ell}$, which has no solution unless $t^{2}=0 \bmod \varpi^{\ell}$.)

This means we can reuse our previous results for $X_{\ell}^{1}$ and $X_{\ell}^{2}$. Let $\Delta_{0}$ be the discriminant of $B_{0}$ and, from now on, $\chi$ the quadratic character associated with $B_{0}$ and $\varepsilon=\chi\left(\Delta_{0}\right)$. 
Proposition. If $n_{0}=0, n_{1}=$ ord $\Delta=1$, and $n=2 k+1$, the correction factor is

$$
\frac{Z(\alpha-k-1)}{Z(2 \alpha-n-1)}
$$

Proof. According to (16) and (17),

$$
\begin{aligned}
& X_{\ell}^{B}\left(t^{2}\right)=q^{-\lceil(\ell-1) / 2\rceil}, \\
& \text { if } t^{2}=0 \bmod \varpi^{\ell} \text {, } \\
& X_{\ell}^{B}\left(t^{2}\right)=0 \text {, } \\
& \text { if } t^{2} \neq 0 \bmod \varpi^{\ell} \text {. }
\end{aligned}
$$

With $z=q^{-\beta}, w=z q^{-1}$, and $u=z^{2} q^{-1}$, it follows that

$$
X^{B}\left(\beta ; t^{2}\right)=\sum_{0 \leq \ell \leq 2 T} z^{\ell} q^{-\lceil(\ell-1) / 2\rceil}=z \sum_{-1 \leq \ell \leq 2 T-1} z^{\ell} q^{-\lceil\ell / 2\rceil},
$$

which, according to (11), simplifies to

$$
\begin{aligned}
X^{B}\left(\beta ; t^{2}\right) & =z\left[\frac{1+w}{1-u}-\frac{w+u}{1-u} u^{T}+z^{-1}-u^{T}\right] \\
& =\frac{1+z}{1-u}-\frac{z+u}{1-u} u^{T}=\frac{1+z}{1-u}\left[1-\frac{u+z}{1+z} u^{T}\right] .
\end{aligned}
$$

This fits the prototype (8) with $v=-z$. With $a=q^{-\alpha}$, the local period (9) is

$$
\frac{Z(\alpha-n)}{1 /(1+a z)}=\frac{Z(\alpha-n)\left(1-a^{2} z^{2}\right)}{1-a z}=\frac{Z(\alpha-n) Z(\alpha-n+k)}{Z(2 \alpha-2 n+2 k)} .
$$

Proposition. If $n_{0}=0, n_{1}=$ ord $\Delta=2$, and $n=2 k+2$, the correction factor is

$$
\frac{Z(2 \alpha-n) Z(\alpha-k-2)}{Z(2 \alpha-n-2)} \text {. }
$$

Proof. According to (16) and (17),

$$
\begin{aligned}
& X_{\ell}^{B}\left(t^{2}\right)=q^{-2\lceil(\ell-1) / 2\rceil}, \quad \text { if } t^{2}=0 \bmod \varpi^{\ell}, \\
& X_{\ell}^{B}\left(t^{2}\right)=0, \quad \text { if } t^{2} \neq 0 \bmod \varpi^{\ell} .
\end{aligned}
$$

With $z=q^{-\beta}$ and $u=z^{2} q^{-2}$, it follows that

$$
X^{B}\left(\beta ; t^{2}\right)=\sum_{0 \leq \ell \leq 2 T} z^{\ell} q^{-2\lceil(\ell-1) / 2\rceil} .
$$

This is the same as in the previous proposition, but with $q^{-2}$ instead of $q^{-1}$ (so, $u=z^{2} q^{-2}$ here corresponds to $u=z^{2} q^{-1}$ there). That is, the local period is

$$
\frac{Z(\alpha-n) Z(\alpha-n+k)}{Z(2 \alpha-2 n+2 k)} \text {. }
$$

Proposition. If $n_{0}=n_{1}=$ ord $\Delta=1$ and $n=2 k+2$, the correction factor is

$$
\frac{Z(2 \alpha-n)}{L(\alpha-k-1, \chi)}
$$

where $\chi$ is the quadratic character associated to $B_{0}$. 
Proof. According to (16), (17) and the proof of proposition (10),

$$
\begin{array}{ll}
X_{\ell}^{B}\left(t^{2}\right)=q^{-\ell}, & \text { if } t^{2}=0 \bmod \varpi^{\ell}, \\
X_{\ell}^{B}\left(t^{2}\right)=X_{\ell}^{1}\left(t^{2}\right) \cdot q^{-T}=(1+\varepsilon) q^{-\ell}, & \text { if } t^{2} \neq 0 \bmod \varpi^{\ell} .
\end{array}
$$

With $z=q^{-\beta}, w=z q^{-1}$, and $u=z^{2} q^{-2}=w^{2}$, it follows that

$$
X^{B}\left(\beta ; t^{2}\right)=\sum_{0 \leq \ell \leq 2 T} z^{\ell} q^{-\ell}+(1+\varepsilon) \sum_{\ell>2 T} z^{\ell} q^{-\ell}=\frac{1}{1-w}+\frac{\varepsilon w}{1-w} u^{T} .
$$

With $a=q^{-\alpha}$, we then obtain

$$
\Pi^{B}(\alpha, \beta)=\frac{1}{1-w}\left[\frac{1}{1-a}+\frac{\varepsilon w}{1-a u}\right]=\frac{(1+\varepsilon w)(1-\varepsilon a w)}{(1-w)(1-a)(1-a u)} .
$$

That is, the local period (7) is

$$
\frac{1-\varepsilon a w}{Z(\alpha) \cdot(1-a)(1-a u)}=\frac{Z(\alpha-n)}{L(\alpha-n+k+1, \chi)} .
$$

Proposition. If $n_{0}=1, n_{1}=$ ord $\Delta=2$, and $n=2 k+3$, the correction factor is

$$
\frac{1}{L(\alpha-k-2, \chi)}
$$

where $\chi$ is the quadratic character associated to $B_{0}$.

Proof. According to (16), (17) and the proof of proposition (10),

$$
\begin{array}{ll}
X_{\ell}^{B}\left(t^{2}\right)=q^{-\ell-\lceil(\ell-1) / 2\rceil}, & \text { if } t^{2}=0 \bmod \varpi^{\ell}, \\
X_{\ell}^{B}\left(t^{2}\right)=X_{\ell}^{1}\left(t^{2}\right) \cdot q^{-2 T}=(1+\varepsilon) q^{-\ell-T}, & \text { if } t^{2} \neq 0 \bmod \varpi^{\ell} .
\end{array}
$$

With $z=q^{-\beta}, w=z q^{-1}$, and $u=z^{2} q^{-3}=w^{2} q^{-1}$, it follows that

$$
\begin{aligned}
X^{B}\left(\beta ; t^{2}\right) & =\sum_{0 \leq \ell \leq 2 T} w^{\ell} q^{-\lceil(\ell-1) / 2\rceil}+(1+\varepsilon) \sum_{\ell>2 T} z^{\ell} q^{-\ell-T} \\
& =\frac{1+w}{1-u}-\frac{w+u}{1-u} u^{T}+\frac{(1+\varepsilon) w}{1-w} u^{T}=\frac{1+w}{1-u}\left[1-\frac{u-\varepsilon w}{1-\varepsilon w} u^{T}\right] .
\end{aligned}
$$

(The first sum is the same as in (18), with $w$ and $u=w^{2} q^{-1}$ here in place of $z$ and $u=z^{2} q^{-1}$ there.) This fits the prototype (8) with $v=\varepsilon w$. With $a=q^{-\alpha}$, the local period (9) is

$$
\frac{Z(\alpha-n)}{1 /(1-\varepsilon a w)}=\frac{Z(\alpha-n)}{L(\alpha-n+k+1, \chi)} .
$$

Proposition. If $n_{0}=2, n_{1}=$ ord $\Delta=1$, and $n=2 k+3$, the correction factor is

$$
\frac{1}{Z(\alpha-k-2)} \text {. }
$$

Proof. According to (16), (17) and the proof of proposition (14) (anisotropic case),

$$
\begin{array}{ll}
X_{\ell}^{B}\left(t^{2}\right)=q^{-\ell-\lceil\ell / 2\rceil}, & \text { if } t^{2}=0 \bmod \varpi^{\ell}, \\
X_{\ell}^{B}\left(t^{2}\right)=X_{\ell}^{1}\left(t^{2}\right) \cdot q^{-T}=\left(1+q^{-1}\right) q^{-\ell-T}, & \text { if } t^{2} \neq 0 \bmod \varpi^{\ell} .
\end{array}
$$


With $z=q^{-\beta}, w=z q^{-1}$, and $u=z^{2} q^{-3}=w^{2} q^{-1}$, we have

$$
\sum_{0 \leq \ell \leq 2 T} z^{\ell} X_{\ell}^{B}\left(t^{2}\right)=\sum_{0 \leq \ell \leq 2 T} w^{\ell} q^{-\lceil\ell / 2\rceil}=\frac{1+w q^{-1}}{1-u}-\frac{w q^{-1}+u}{1-u} u^{T},
$$

according to (11) (with $w, w q^{-1}$, and $u=w^{2} q^{-1}$ here in place of $z, w=z q^{-1}$, and $u=z^{2} q^{-1}$ there). We have also

$$
\sum_{\ell>2 T} z^{\ell} X_{\ell}^{B}\left(t^{2}\right)=\left(1+q^{-1}\right) q^{-T} \sum_{\ell>2 T} w^{\ell}=\frac{\left(1+q^{-1}\right) w}{1-w} u^{T}
$$

leading to

$$
\begin{aligned}
X^{B}\left(\beta ; t^{2}\right) & =\frac{1+w q^{-1}}{1-u}-\frac{w q^{-1}+u}{1-u} u^{T}+\frac{w+w q^{-1}}{1-w} u^{T} \\
& =\frac{1+w q^{-1}}{1-u}\left[1-\frac{u-w}{1-w} u^{T}\right] .
\end{aligned}
$$

This fits the prototype (8) with $v=w$. With $a=q^{-\alpha}$, the local period (9) is

$$
\frac{Z(\alpha-n)}{1 /(1-a w)}=\frac{Z(\alpha-n)}{Z(\alpha-n+k+1)} \text {. }
$$

Proposition. If $n_{0}=n_{1}=$ ord $\Delta=2$ and $n=2 k+4$, the correction factor $i s$

$$
\frac{Z(2 \alpha-n)}{Z(\alpha-k-3)} \text {. }
$$

Proof. According to (16), (17) and the proof of proposition (14) (anisotropic case),

$$
\begin{aligned}
& X_{\ell}^{B}\left(t^{2}\right)=q^{-2 \ell}, \quad \text { if } t^{2}=0 \bmod \varpi^{\ell}, \\
& X_{\ell}^{B}\left(t^{2}\right)=X_{\ell}^{1}\left(t^{2}\right) \cdot q^{-2 T}=\left(1+q^{-1}\right) q^{-\ell-2 T}, \quad \text { if } t^{2} \neq 0 \bmod \varpi^{\ell} \text {. }
\end{aligned}
$$

With $z=q^{-\beta}, w=z q^{-1}$, and $u=z^{2} q^{-4}=w^{2} q^{-2}$, we have

$$
\begin{aligned}
X^{B}\left(\beta ; t^{2}\right) & =\sum_{0 \leq \ell \leq 2 T} z^{\ell} q^{-2 \ell}+\left(1+q^{-1}\right) \sum_{\ell>2 T} z^{\ell} q^{-\ell-2 T} \\
& =\frac{1-w q^{-1} u^{T}}{1-w q^{-1}}+\frac{w+w q^{-1}}{1-w} u^{T}=\frac{1}{1-w q^{-1}}\left[1-\frac{u-w}{1-w} u^{T}\right] .
\end{aligned}
$$

This fits the prototype (8) with $v=w$. With $a=q^{-\alpha}$, the local period (9) is

$$
\frac{Z(\alpha-n)}{1 /(1-a w)}=\frac{Z(\alpha-n)}{Z(\alpha-n+k+1)} \text {. }
$$

\section{The General CASE}

We resume the discussion and the notation from section 1 . We saw that

$$
\left(E_{\varphi}, F\right)_{H}=(\eta, F)_{\Theta} \cdot \int_{\Theta_{\mathbb{A}} \backslash H_{\mathbb{A}}} \varphi_{\omega} \cdot f
$$

where $f$ is a spherical vector in $\operatorname{Ind}_{\Theta}^{H} 1$ normalized by $f(1)=1$. We want to express this global integral as a product of local integrals.

If $F$ generates an irreducible representation, so does $f$ generate an irreducible representation $\pi=\otimes_{v} \pi_{v}$. If the period is nonzero, then each $\pi_{v}$ is a quotient of a degenerate unramified principal series with respect to the Levi component $M^{Q}$ and 
with character $m_{\lambda} \mapsto|\lambda|^{\beta_{v}}$. Let $f_{v}$ be a generator of $\pi_{v}$ normalized by $f_{v}(1)=1$. The intertwining between models of $\pi$ takes $f$ to a multiple of $\otimes_{v} f_{v}$, so

$$
\int_{\Theta_{\mathbb{A} \backslash H_{\AA}}} \varphi_{\omega} \cdot f=C_{f} \cdot \prod_{v} \int_{\Theta_{v} \backslash H_{v}} \varphi_{\omega, v} \cdot f_{v}
$$

for some constant $C_{f}$.

We now work locally and suppress $v$. Let $\psi_{\beta}$ be the principal series; in particular, $\psi_{\beta}\left(m_{\lambda}\right)=|\lambda|^{\beta}$. The function $f$ generates a model of $\pi$ consisting of left $\Theta$ - and right $K$-invariant functions. The restriction $\operatorname{Res}_{Q}^{H} \pi$ may be modelled by the very same functions. Thus, it is irreducible too and isomorphic to the principal series representation. But we may model both of them with left $\Theta \cap Q-$ and right $K_{-}$ invariant functions and $f(1)=\psi_{\beta}(1)$; therefore, $\psi_{\beta}$ is the restriction of $f$ to $Q$.

(19) Proposition. The local integral at odd primes is given by

$$
\int_{\Theta \backslash H} \varphi_{\omega} \cdot f=\frac{\operatorname{vol}(\Theta \backslash \Theta K) \cdot \Pi^{n}(\alpha-\beta-n, \beta)}{Z(\alpha) \cdot X^{n}(0 ; 1)} .
$$

Proof. This is the same computation we did in the proof of proposition (3); by the exact same reasoning and with the same notation as there, the local integral is

$$
\frac{\operatorname{vol}(\Theta \backslash \Theta K)}{Z(\alpha) \cdot X^{n}(0 ; 1)} \int_{(\Theta \cap Q) \backslash Q} \varphi_{\omega} \cdot f=\frac{\operatorname{vol}(\Theta \backslash \Theta K)}{Z(\alpha) \cdot X^{n}(0 ; 1)} \int_{(\Theta \cap Q) \backslash Q} \varphi_{\omega} \cdot|\lambda|^{\beta} .
$$

But that last integral is the very same one computed from (5) onward.

\section{Appendix A. The COnstant term}

The functional equation of an Eisenstein series is inherited by its periods. As the equation involves the constant term, we describe briefly how that term may be obtained using the methods we have used so far.

With $\operatorname{vol}\left(N_{k} \backslash N_{\mathbb{A}}\right)=1$, the constant term of $E_{s}$ is

$$
c E_{s}(m)=\varphi^{\circ}(m)+\int_{N_{\mathrm{A}}} \varphi^{\circ}(\xi n m) \mathrm{d} n=|\lambda|^{\alpha}+\int_{N_{\mathrm{A}}} \varphi^{\circ}(\xi n m) \mathrm{d} n,
$$

where $\xi$ is the long Weyl element in $P \backslash G / P$. The second summand factors over primes.

At a place $v$ (suppressed from this point onward), express the form on $k^{n+3}=$ $\left(k \cdot e^{\prime}\right) \oplus k^{n+1} \oplus(k \cdot e)$ by

$$
\left(\begin{array}{lll} 
& & 1 \\
& B &
\end{array}\right)
$$

and write

$$
n=\left(\begin{array}{ccc}
1 & a & -\frac{1}{2} B(a) \\
& \text { id } & * \\
& & 1
\end{array}\right) \quad \text { and } \quad m=\left(\begin{array}{ccc}
\lambda & & \\
& \theta & \\
& & 1 / \lambda
\end{array}\right)
$$


with $\theta \in \Theta=\mathrm{O}(B)$. We have

$$
\begin{aligned}
\int_{N} \varphi(\xi n m) \mathrm{d} n & =\int_{N} \int_{k^{\times}}|t|^{\alpha} \Phi(t e \cdot \xi n m) \mathrm{d} t \mathrm{~d} n=\int_{N} \int_{k^{\times}}|t|^{\alpha} \Phi\left(t e^{\prime} \cdot n m\right) \mathrm{d} t \mathrm{~d} n \\
& =\int_{N} \int_{k^{\times}}|t|^{\alpha} \Phi\left(t e^{\prime} \cdot m n\right) \mathrm{d} t \cdot \delta_{P}(m) \mathrm{d} n \\
& =\int_{N} \int_{k^{\times}}|t|^{\alpha}|\lambda|^{n+1} \Phi\left(\lambda t e^{\prime} \cdot n\right) \mathrm{d} t \mathrm{~d} n .
\end{aligned}
$$

At this point, we disregard the normalization of the integral, and proceed.

$$
\begin{aligned}
\int_{N} \varphi(\xi n m) \mathrm{d} n & =|\lambda|^{(n+1)-\alpha} \cdot \int_{k^{\times}}|t|^{\alpha} \int_{N} \Phi\left(t e^{\prime} \cdot n\right) \mathrm{d} n \mathrm{~d} t \\
& =|\lambda|^{(n+1)-\alpha} \cdot \int_{k^{\times}}|t|^{\alpha} \int_{k^{n+1}} \Phi\left(t, a t,-\frac{1}{2 t} B(a t)\right) \mathrm{d} a \mathrm{~d} t \\
& =|\lambda|^{(n+1)-\alpha} \int_{k^{\times}}|t|^{\alpha-(n+1)} \int_{k^{n+1}} \Phi\left(t, a,-\frac{1}{2 t} B(a)\right) \mathrm{d} a \mathrm{~d} t .
\end{aligned}
$$

Using the same notation we used before

$$
\int_{N} \varphi(\xi n m) \mathrm{d} n=|\lambda|^{(n+1)(1-s)} \cdot X^{n+1}(\alpha-(n+1) ; 0) .
$$

Therefore, recalling $\alpha=(n+1) s$,

$$
\int_{N} \varphi^{\circ}(\xi n m) \mathrm{d} n=|\lambda|^{(n+1)(1-s)} \cdot \frac{X^{n+1}(-(n+1)(1-s) ; 0)}{Z((n+1) s)} .
$$

With the conventions of the strategy overview at the end of section 2 ,

$$
X(\beta ; 0)=\lim _{a \rightarrow 1}\left((1-a) \sum_{T \geq 0} a^{T} X\left(\beta ; \varpi^{2 T}\right)\right)=-\operatorname{Res}_{a=1} R(z, w, u, a) .
$$

\section{REFERENCES}

[1] Avraham Aizenbud, Dmitry Gourevitch, Steve Rallis, and Gérard Schiffmann, Multiplicity one theorems, Ann. of Math. (2) 172 (2010), no. 2, 1407-1434. MR2680495 (2011g:22024)

[2] Avraham Aizenbud, Dmitry Gourevitch, and Eitan Sayag, $(O(V \oplus F), O(V))$ is a Gelfand pair for any quadratic space $V$ over a local field F, Math. Z. 261 (2009), no. 2, 239-244. MR2457297 (2010a:22020)

[3] J. W. S. Cassels, Rational quadratic forms, London Mathematical Society Monographs, vol. 13, Academic Press Inc., London, 1978. MR522835 (80m:10019)

[4] Paul Garrett, Euler factorization of global integrals, NSF-CBMS Regional Conference in Mathematics on Euler Products and Eisenstein Series (Texas Christian University, Fort Worth, Tex., May 20-24, 1996), Automorphic forms, automorphic representations, and arithmetic (Robert S. Doran, Ze-Li Dou, and George T. Gilbert, eds.), Proc. Sympos. Pure Math., vol. 66.2, Amer. Math. Soc., Providence, R.I., 1999, pp. 35-101. MR1703758 (2000m:11043)

[5] Stephen Gelbart, Ilya Piatetski-Shapiro, and Stephen Rallis, Explicit constructions of automorphic L-functions, Lecture Notes in Mathematics, vol. 1254, Springer-Verlag, Berlin, 1987. MR892097 (89k:11038)

[6] David Ginzburg, Dihua Jiang, and David Soudry, Poles of L-functions and theta liftings for orthogonal groups, J. Inst. Math. Jussieu 8 (2009), no. 4, 693-741. MR2540878

[7] D. Ginzburg, I. Piatetski-Shapiro, and S. Rallis, L functions for the orthogonal group, Mem. Amer. Math. Soc. 128 (1997), no. 611. MR1357823 (98m:11041)

[8] David Ginzburg, Stephen Rallis, and David Soudry, Periods, poles of L-functions and symplectic-orthogonal theta lifts, J. Reine Angew. Math. 487 (1997), 85-114. MR1454260 (98f:11046) 
[9] Benedict H. Gross, Some applications of Gelfand pairs to number theory, Bull. Amer. Math. Soc. (N.S.) 24 (1991), no. 2, 277-301. MR1074028 (91i:11055)

[10] Benedict H. Gross and Dipendra Prasad, Test vectors for linear forms, Math. Ann. 291 (1991), no. 2, 343-355. MR1129372 (92k:22028)

[11] On the decomposition of a representation of $\mathrm{SO}_{n}$ when restricted to $\mathrm{SO}_{n-1}$, Canad. J. Math. 44 (1992), no. 5, 974-1002. MR1186476 (93j:22031)

[12] On irreducible representations of $\mathrm{SO}_{2 n+1} \times \mathrm{SO}_{2 m}$, Canad. J. Math. 46 (1994), no. 5, 930-950. MR1295124 (96c:22028)

[13] Benedict H. Gross and Mark Reeder, From Laplace to Langlands via representations of orthogonal groups, Bull. Amer. Math. Soc. (N.S.) 43 (2006), no. 2, 163-205. MR2216109 (2007a:11159)

[14] Atsushi Ichino and Tamotsu Ikeda, On the periods of automorphic forms on special orthogonal groups and the Gross-Prasad conjecture, Geom. Funct. Anal. 19 (2010), no. 5, 1378-1425. MR2585578

[15] Jun-ichi Igusa, An introduction to the theory of local zeta functions, AMS/IP Studies in Advanced Mathematics, vol. 14, Amer. Math. Soc., Providence, R.I., 2000. MR1743467 (2001j:11112)

[16] H. Iwaniec and P. Sarnak, Perspectives on the analytic theory of L-functions, Visions in Mathematics, towards 2000 (Tel Aviv University, August 25-September 3, 1999), GAFA 2000 (N. Alon, J. Bourgain, A. Connes, M. Gromov, and V. Milman, eds.), Birkhäuser, Basel, 2000, pp. 705-741. Geom. Funct. Anal., Special Volume, Part II. MR1826269 (2002b:11117)

[17] Hervé Jacquet, Erez Lapid, and Jonathan Rogawski, Periods of automorphic forms, J. Amer. Math. Soc. 12 (1999), no. 1, 173-240. MR1625060 (99c:11056)

[18] H. Jacquet and J. A. Shalika, On Euler products and the classification of automorphic representations. I, Amer. J. Math. 103 (1981), no. 3, 499-558. MR618323 (82m:10050a)

[19] - On Euler products and the classification of automorphic forms. II, Amer. J. Math. 103 (1981), no. 4, 777-815. MR623137 (82m:10050b)

[20] Dihua Jiang, Periods of automorphic forms, Proceedings of the International Conference on Complex Geometry and Related Fields (Stephen S.-T. Yau, Zhijie Chen, Jianpan Wang, and Sheng-Li Ten, eds.), AMS/IP Stud. Adv. Math., vol. 39, Amer. Math. Soc., Providence, R.I., 2007, pp. 125-148. MR2338623 (2008k:11052)

[21] Dihua Jiang and David Soudry, The local converse theorem for $\mathrm{SO}(2 n+1)$ and applications, Ann. of Math. (2) 157 (2003), no. 3, 743-806. MR1983781 (2005b:11193)

[22] — Generic representations and local Langlands reciprocity law for p-adic $\mathrm{SO}_{2 n+1}$, Contributions to automorphic forms, geometry, and number theory (Haruzo Hida, Dinakar Ramakrishnan, and Freydoon Shahidi, eds.), Johns Hopkins Univ. Press, Baltimore, Md., 2004, pp. 457-519. MR2058617 (2005f:11272)

[23] Shin-ichi Kato, Atsushi Murase, and Takashi Sugano, Whittaker-Shintani functions for orthogonal groups, Tohoku Math. J. (2) 55 (2003), no. 1, 1-64. MR1956080 (2003m:22020)

[24] Henry H. Kim, On local L-functions and normalized intertwining operators, Canad. J. Math. 57 (2005), no. 3, 535-597. MR2134402 (2006a:11063)

[25] Erez Lapid and Omer Offen, Compact unitary periods, Compos. Math. 143 (2007), no. 2, 323-338. MR2309989 (2008g:11091)

[26] Erez Lapid and Jonathan Rogawski, Periods of Eisenstein series, C. R. Acad. Sci. Paris Sér. I Math. 333 (2001), no. 6, 513-516. MR1860921 (2002k:11072)

[27] Atsushi Murase and Takashi Sugano, Shintani function and its application to automorphic L-functions for classical groups. I. The case of orthogonal groups, Math. Ann. 299 (1994), no. 1, 17-56. MR1273075 (96c:11054)

[28] O. Timothy O'Meara, Introduction to quadratic forms, Classics in Mathematics, SpringerVerlag, Berlin, 2000. Reprint of the 1973 edition. MR1754311 (2000m:11032)

[29] I. I. Pjateckij-Šapiro, Euler subgroups, Summer School on Group Representations of the Bolyai János Mathematical Society (Budapest, August 16-September 3, 1971), Lie groups and their representations (I. M. Gelfand, ed.), John Wiley \& Sons, New York, 1975, pp. 597-620. MR0406935 (53 \#10720)

[30] Vladimir Platonov and Andrei Rapinchuk, Algebraic groups and number theory, Pure and Applied Mathematics, vol. 139, Academic Press Inc., Boston, Mass., 1994. Translated from the 1991 Russian original by Rachel Rowen. MR1278263 (95b:11039) 
[31] Stephen Rallis, L-functions and the oscillator representation, Lecture Notes in Mathematics, vol. 1245, Springer-Verlag, Berlin, 1987. MR887329 (89b:11046)

[32] Winfried Scharlau, Quadratic and Hermitian forms, Grundlehren der Mathematischen Wissenschaften, vol. 270, Springer-Verlag, Berlin, 1985. MR770063 (86k:11022)

[33] Binyong Sun and Chen-Bo Zhu, Multiplicity one theorems: the archimedean case, Ann. of Math. (2), to appear, available at arXiv:0903.1413v2 [math.RT] .

[34] André Weil, Adeles and algebraic groups, Progress in Mathematics, vol. 23, Birkhäuser, Boston, Mass., 1982. MR670072 (83m:10032)

Departamento de Matemática, Instituto Superior Técnico, Universidade Técnica de Lisboa, Av. Rovisco Pais, 1049-001 Lisboa, Portugal

E-mail address: joao.boavida@ist.utl.pt 